\title{
Confiabilidade da Declaração de Nascido Vivo como fonte de informação sobre os defeitos congênitos no Município de São Carlos, São Paulo, Brasil
}

\author{
Reliability of birth certificates as a source of information \\ on congenital defects in the City of São Carlos, São Paulo, Brazil
}

Geiza César Nhoncanse ${ }^{1}$ Débora Gusmão Melo ${ }^{1}$
${ }^{1}$ Departamento de Medicina, Universidade Federal de São Carlos. Rod. Washington Luís (SP-310), Km 235, Campus da UFSCar, Departamento de Medicina (área norte). 13565-905 São Carlos SP. dgmelo@ufscar.br

\begin{abstract}
The reliability of the information on congenital defects listed in birth certificates of the Live Birth Information System (SINASC) in the City of São Carlos, São Paulo, Brazil, was evaluated. A descriptive study that reviewed all 15,249 birth certificates from 2003 to 2007 compared the data with information from medical records and death certificates. Errors in accuracy and precision, mainly related to the description of the anomaly when it was transcribed from medical records to the birth certificates, in addition to coding and the input to SINASC, diminished the reliability of the birth certificates as a source of information on congenital defects. The results suggested that the involvement of the Municipal Health Department is essential to improve SINASC, because this is the location of the coding and input system, and training of the professionals who fill out the birth certificates. With guidance on the importance and function of the birth certificates, SINASC can become an excellent monitoring and surveillance system for congenital defects.
\end{abstract}

Key words Information systems, Birth certificates, Congenital defects
Resumo Avaliou-se a confiabilidade das informações sobre defeitos congênitos presentes nas Declarações de Nascido Vivo (DNV) do Sistema de Informações sobre Nascidos Vivos (SINASC) no Município do São Carlos, São Paulo, Brasil, por meio de estudo descritivo que revisou todas as 15.249 DNV, de 2003 a 2007, e as comparou com as informações de prontuários e de declarações de óbito. Erros de acurácia e precisão, relacionados principalmente à transcrição da descrição da anomalia do prontuário para a DNV, e a codificação $e$ a alimentação do SINASC, comprometeram a confiabilidade das DNV como fonte de informações sobre os defeitos congênitos. Os resultados sugerem que para aprimoramento do SINASC, é fundamental o envolvimento das Secretarias Municipais de Saúde, visto ser esse o local de codificação e de alimentação do sistema, e também a capacitação dos profissionais que preenchem a DNV. Com orientação sobre a importância e o funcionamento da DNV, o SINASC pode ser tornar um excelente sistema de monitoramento $e$ vigilância para os defeitos congênitos.

Palavras-chave Sistemas de informação, Declaração de nascido vivo, Defeitos congênitos 


\section{Introdução}

A Declaração de Nascido Vivo (DNV) é um documento padronizado pelo Ministério da Saúde, preenchido em todo o território nacional, para todas as crianças nascidas em estabelecimentos de saúde ou domicílio, imprescindível para o registro civil. Ela é o documento de entrada do Sistema de Informações sobre Nascidos Vivos (SINASC), que organiza e fornece informações sobre os nascimentos para os gestores da saúde em todos os níveis. A DNV é impressa em três vias previamente numeradas através do Centro $\mathrm{Na}$ cional de Epidemiologia (CENEPI) e da Fundação Nacional de Saúde; cada via possui uma cor distinta (branca, amarela e rosa). O documento é preenchido nos estabelecimentos de saúde ou nos cartórios de registro civil (nos casos dos partos domiciliares sem assistência de saúde imediata), sendo a via branca devolvida à Secretaria Municipal de Saúde, a via amarela entregue aos pais do recém-nascido e a via rosa anexada ao prontuário do recém-nascido no estabelecimento de saúde. Os dados coletados por meio das DNV são consolidados nas Secretarias Municipais de Saúde, que devem encaminhá-los mensalmente às Secretarias Estaduais. Nas Secretarias Estaduais de Saúde, as informações são processadas por município e criticadas, para serem enviadas ao Ministério da Saúde, que as publica por meio do Departamento de Informática do Sistema Único de Saúde (DATASUS) ${ }^{1}$.

A partir de 2000, o Brasil adotou uma nova versão da DNV na qual consta o Campo 34, que registra a presença ou ausência de "malformação congênita e/ou anomalia cromossômica". Malformação congênita é definida como uma anomalia estrutural presente ao nascimento, resultado de um desenvolvimento intrinsecamente anormal. A expressão "defeito congênito" contempla uma definição mais ampla, que inclui toda anomalia funcional ou estrutural, decorrente de fator originado antes do nascimento, seja genético, ambiental ou desconhecido, mesmo quando o defeito não for aparente no recém-nascido e só se manifestar mais tarde. Portanto, do ponto de vista biológico, os defeitos congênitos (DC) representam um grupo heterogêneo de distúrbios do desenvolvimento embriofetal, com fatores etiológicos distintos, muitas vezes simultaneamente envolvidos. É possível identificar etiologia genética, cromossômica ou gênica em cerca de 20 a 30\% dos DC. Situações nas quais há componente ambiental ou interação gene-ambiente são mais numerosas e difíceis de serem reconheci- das, de forma que aproximadamente $60 \%$ dos DC permanecem sem etiologia definida ${ }^{2}$.

A incidência geral dos DC no Brasil não difere daquela encontrada em outras regiões do mundo e, de modo geral, 2 a 5\% dos recém-nascidos brasileiros apresentam algum DC. Neste contexto, os DC surgem como um problema de saúde pública e requerem atenção especial. Principalmente, quando se considera que à medida que os indicadores de saúde da população melhoram, e as doenças causadas por má nutrição, condições insalubres e patógenos exógenos são controladas, uma proporção crescente dos óbitos entre as crianças é atribuída às doenças genéticas e aos DC ${ }^{3-5}$. É o que acontece no Brasil, onde, desde 2005, essas afecções, representadas pelo capítulo XVII da Classificação Internacional de Doenças (CID-10) - "malformações congênitas, deformidades e anomalias cromossômicas" - são a segunda causa de mortalidade infantil em todas as regiões do país ${ }^{6}$.

A introdução do Campo 34 na DNV é um marco importante para a genética comunitária do país. Até então, as informações sobre os DC eram obtidas somente a partir das Declarações de Óbito (DO), cujos dados são organizados pelo Sistema de Informação de Mortalidade (SIM). Com a nova DNV, o SINASC passou a ser um importante instrumento de monitoramento populacional dos $\mathrm{DC}^{4,7-13}$. Para que sistemas de informações, como o SINASC e o SIM, sejam efetivos no monitoramento e no planejamento de ações em saúde pública, a informação divulgada precisa ser confiável. Isso só é possível com o preenchimento correto dos instrumentos de coleta e o adequado fluxo de dados através dos diferentes níveis do sistema.

Analisando as informações do SINASC do Município de São Carlos, São Paulo, publicadas pelo DATASUS, observa-se uma subnotificação dos DC. A cidade de São Carlos está localizada no centro geográfico do Estado de São Paulo, possui cerca de 220.000 habitantes e um índice de desenvolvimento humano de $0,841^{14}$. Até 2008, o Município de São Carlos possuía duas maternidades; uma delas, a Maternidade da Santa Casa de Misericórdia, era responsável por cerca de $80 \%$ dos partos. Em função da taxa de natalidade do município (cerca de 3.000 crianças/ano), são esperados 60 a 150 recém-nascidos com DC por ano. No entanto, de acordo com o DATASUS, no período entre 2003 e 2007, há registro de apenas 17 crianças nascidas com DC, e em 465 casos o Campo 34 da DNV foi assinalado como "ignorado" . A taxa de mortalidade infantil de São 
Carlos é uma das mais baixas do Estado de São Paulo, com média de 8,5 óbitos por mil nascidos vivos entre os anos de 2005 e $2009^{15}$. Analisando as informações do SIM, observa-se que o capítulo XVII da CID-10 representou a segunda causa de óbito infantil entre os anos de 2003 e 2007, com 39 crianças falecidas devido a doenças genéticas ou DC $(22,29 \%$ de todos os óbitos infantis $)^{6}$. A comparação entre os dados do SINASC e do SIM indica que morreram mais crianças com DC do que nasceram, paradoxo explicado pelo subregistro dos defeitos congênitos nas DNV. Levantou-se a hipótese de que recém-nascidos com DC tiveram o Campo 34 de suas DNV assinalado como "ignorado" ou não preenchido (sem informação). Nesse trabalho, procurou-se avaliar a confiabilidade da DNV como fonte de informação dos DC no Município de São Carlos.

\section{Metodologia}

Realizou-se estudo descritivo, por meio de revisão de todas as DNV de 2003 a 2007, arquivadas em papel (via branca) na Vigilância Epidemiológica (VE) do Município de São Carlos, totalizando 15.249 DNV. Adicionalmente, revisou-se as DO, também arquivadas na VE, de crianças menores de um ano, falecidas em São Carlos entre 2003 e 2007, cuja declaração mencionava o capítulo XVII da CID-10, totalizando 47 DO. Por fim, revisou-se os prontuários das crianças nascidas na Santa Casa de Misericórdia de São Carlos, cujo Campo 34 da DNV foi assinalado como "sim", "ignorado" ou não preenchido, ou cuja DO havia sido revisada, totalizando 140 prontuários. As informações obtidas a partir dos três documentos (DNV, DO e prontuário) foram comparadas com as informações publicadas pelo
DATASUS e entre si. Para a avaliação da concordância dos três documentos em relação à presença ou ausência de DC, foi utilizado o índice kappa (k), calculado com auxílio do programa Statistical Analyses System (SAS, versão 8.01), tendo como nível de significância o valor de 0,05 e o intervalo de confiança de $95 \%$. A pesquisa foi aprovada pelo Comitê de Ética em Pesquisa com Seres Humanos da UFSCar.

\section{Resultados}

Foram identificadas incongruências entre os dados das DNV arquivadas na VE e os publicados pelo SINASC. De acordo com o DATASUS, no período de 2003 a 2007 nasceram 17 crianças com DC e, em 465 casos, o Campo 34 da DNV foi assinalado como "ignorado". No entanto, para esse mesmo período, os dados arquivados na VE apontam o nascimento de 23 crianças com DC, em apenas 2 casos o Campo 34 foi assinalo como "ignorado" e, em 124 casos, não havia informação sobre os DC, pois nenhuma das três opções possíveis ("sim”, “não” ou "ignorado”) foi assinalada no Campo 34 da DNV; ou seja, em 124 casos não houve completeza em relação ao Campo 34 da DNV. A não completeza variou de 1,35\% em 2003 a 0,43\% em 2007, com média de $0,82 \%$ entre 2003 e 2007 (Tabela 1).

Quanto às DO, entre 2003 e 2007, o DATASUS registra 39 óbitos infantis em decorrência de patologias do capítulo XVII da CID-10, mas na VE foram encontradas 47 DO com essa característica, das quais 41 crianças nasceram no Município de São Carlos entre 2003 e 2007 (2 crianças nasceram em 2002 e faleceram em 2003, 4 nasceram em outros municípios e faleceram em São Carlos; o que justificou a exclusão desses 6 casos da

Tabela 1. Comparação entre os dados sobre defeitos congênitos do SINASC arquivados em papel na VE e publicados pelo DATASUS, para o total de nascimentos ocorridos nos anos de 2003 a 2007, em São Carlos, Brasil.

\begin{tabular}{|c|c|c|c|c|c|c|c|c|c|c|c|c|c|c|c|c|c|}
\hline & \multicolumn{8}{|c|}{ VE } & \multicolumn{8}{|c|}{ DATASUS } & \multirow{3}{*}{$\begin{array}{c}\text { Total } \\
\text { nasc. } \\
\mathrm{N}\end{array}$} \\
\hline & \multicolumn{2}{|c|}{ Sim } & \multicolumn{2}{|c|}{ Não } & \multicolumn{2}{|c|}{ Ignorado } & \multicolumn{2}{|c|}{$\begin{array}{c}\text { Sem } \\
\text { informação }\end{array}$} & \multicolumn{2}{|c|}{ Sim } & \multicolumn{2}{|c|}{ Não } & \multicolumn{2}{|c|}{ Ignorado } & \multicolumn{2}{|c|}{$\begin{array}{c}\text { Sem } \\
\text { informação }\end{array}$} & \\
\hline & $\mathbf{N}$ & $\%$ & $\mathbf{N}$ & $\%$ & $\mathbf{N}$ & $\%$ & $\mathbf{N}$ & $\%$ & $\mathbf{N}$ & $\%$ & $\mathbf{N}$ & $\%$ & $\mathbf{N}$ & $\%$ & $\mathbf{N}$ & $\%$ & \\
\hline 2003 & 4 & 0,13 & 2.930 & 98,52 & 0 & 0 & 40 & 1,35 & 3 & 0,10 & 2.885 & 97,00 & 86 & 2,90 & - & - & 2.974 \\
\hline 2004 & 4 & 0,13 & 2.986 & 99,20 & 0 & 0 & 20 & 0,67 & 4 & 0,13 & 2.669 & 88,67 & 337 & 11,20 & - & - & 3.010 \\
\hline 2005 & 6 & 0,19 & 3.017 & 98,88 & 1 & 0,03 & 27 & 0,90 & 5 & 0,16 & 3.012 & 98,72 & 34 & 1,12 & - & - & 3.051 \\
\hline 2006 & 5 & 0,16 & 3.059 & 99,06 & 0 & 0 & 24 & 0,78 & 5 & 0,16 & 3.082 & 99,80 & 1 & 0,04 & - & - & 3.088 \\
\hline 2007 & 4 & 0,12 & 3.108 & 99,42 & 1 & 0,03 & 13 & 0,43 & 0 & 0 & 3.119 & 99,77 & 7 & 0,23 & - & - & 3.126 \\
\hline $2003-2007$ & 23 & 0,15 & 15.100 & 99,02 & 2 & 0,01 & 124 & 0,82 & 17 & 0,11 & 14.767 & 96,84 & 465 & 3,05 & - & - & 15.249 \\
\hline
\end{tabular}


análise). Das 41 crianças são-carlenses, falecidas com DC, em 32 casos (78,05\%) não havia registro do defeito na DNV: em 31 situações o Campo 34 da DNV foi marcado como "não" e em 1 situação não foi preenchido (Tabela 2).

Dos 140 prontuários revisados, 55 (39,29\%) apresentavam descrição de algum DC, sendo que a maior parte desses defeitos (51/55 casos, ou $92,7 \%)$ havia sido detectada pelos SINASC ou SIM. Além dos 23 casos de DC registrados nas DNV e dos 32 casos identificados nas DO, mas não registrados nas DNV, apenas outros 4 casos foram identificados exclusivamente pela revisão dos prontuários; em todas essas quatro situações, o recém-nascido possuía algum DC, mas o Campo 34 da sua DNV não havia sido preenchido. Dessa forma, o cruzamento dos três documentos revisados (DNV, DO e prontuário médico), permitiu identificar 59 crianças nascidas com DC, no período de 2003 a 2007, em São Carlos.

A análise dos três documentos e a comparação com os dados publicados pelo SINASC explicitou dois problemas referentes à acurácia em relação às DNV como fonte de informação sobre os DC: (1) há simplificação da descrição dos DC nas DNV, quando comparadas às descrições dos prontuários e das DO, especialmente nas situações nas quais os recém-nascidos possuem múltiplos defeitos; (2) no processo de codificação e digitação das DNV, realizado no Centro de
Vigilância Epidemiológica do município, somente um único defeito, codificado com base na CID10 , pode ser escolhido para ser repassado à Secretaria Estadual de Saúde, o que obriga o codificador a fazer uso de algum critério pessoal de seleção nos casos com DC múltiplos.

O prontuário foi mais sensível para o registro de DC que a DNV. Dos 59 casos identificados, apenas $4(6,78 \%)$ não foram registrados nos prontuários, enquanto $36(61,02 \%)$ tiveram o Campo 34 da DNV assinalado equivocadamente como "não" ou não informado. A análise dos prontuários e das DO mostrou alto índice de concordância de informações, pois somente 3 dos 41 casos de DC registrados nas DO (7,32\%) não foram descritos nos prontuários. Por outro lado, a comparação entre DO e DNV confirmou a subnotificação dos DC nas DNV, pois apenas 9 crianças $(21,95 \%)$ que faleceram com anomalias haviam sido previamente identificadas pelas DNV (Figura 1). Ou seja, existem muitos falsos negativos em relação ao Campo 34 das DNV no Município de São Carlos, pois parte considerável dos casos de DC não é registrada nas DNV, denotando erros de precisão. Os cruzamentos entre os resultados da revisão dos três documentos são apresentados na Tabela 3. O nível de concordância foi desprezível entre todos os instrumentos, seja na comparação dois a dois, seja na comparação dos três documentos simultaneamente.

Tabela 2. Comparação entre os dados sobre defeitos congênitos registrados nas DNV e nas DO de menores de um ano, nos anos de 2003 a 2007, em São Carlos, Brasil.

Defeito congênito

(DO - capítulo XVII da CID-10)

\begin{tabular}{|c|c|c|c|c|c|c|c|}
\hline & & 2003 & 2004 & 2005 & 2006 & 2007 & 2003-2007 \\
\hline \multirow{6}{*}{ 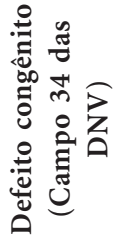 } & Sim & 2 & 3 & 2 & 1 & 1 & 9 \\
\hline & Não & 5 & 6 & 6 & 6 & 8 & 31 \\
\hline & Sem informação (não preenchido) & 0 & 0 & 1 & 0 & 0 & 1 \\
\hline & Nasc. outro município (DNV indisponível) & 1 & 1 & 2 & 0 & 0 & 4 \\
\hline & Nasc. em 2002 (DNV não revisada) & 2 & - & - & - & - & 2 \\
\hline & Total & 10 & 10 & 11 & 7 & 9 & 47 \\
\hline
\end{tabular}

Tabela 3. Concordância entre os três documentos utilizados para registro dos defeitos congênitos, nos anos de 2003 a 2007, em São Carlos, Brasil.

\begin{tabular}{lcccc}
\hline \multicolumn{1}{c}{ Documentos } & Valor kappa & IC em $\mathbf{9 5 \%}$ & Valor $\boldsymbol{p}$ & Concordância \\
\hline DNV x DO & $-0,548$ & $-0,338$ a $-0,759$ & Não é interpretável & Desprezível \\
DNV x prontuário & $-0,071$ & 0,0080 a $-0,15$ & Não é interpretável & Desprezível \\
DO x prontuário & $-0,096$ & 0,069 a $-0,261$ & Não é interpretável & Desprezível \\
DNV x DO x prontuário & $-0,308$ & $-0,161$ a $-0,455$ & Não é interpretável & Desprezível \\
\hline
\end{tabular}




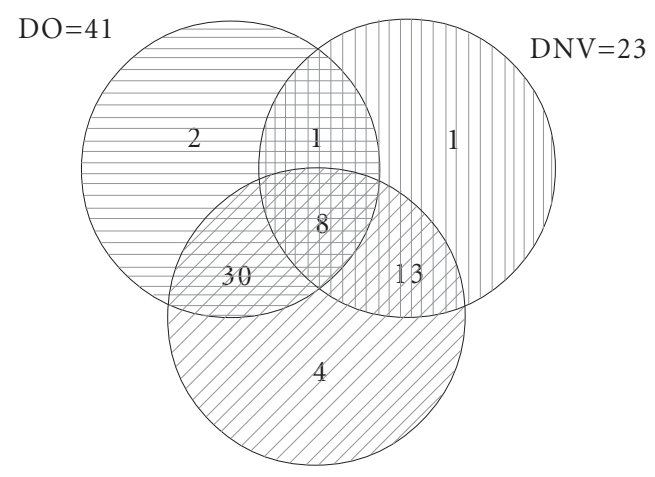

Prontuário $=55$

Figura 1. Cruzamento dos três documentos utilizados para registro dos defeitos congênitos, com identificação de um total de 59 casos, nos anos de 2003 a 2007, em São Carlos, Brasil.

Os valores de kappa negativos indicam que as concordâncias de classificação são piores do que se a classificação em relação à presença ou à ausência de DC fosse feita ao acaso.

\section{Discussão}

A existência de certificados de nascimento, semelhantes às DNV, é comum nos Estados Unidos (chamados "birth certificates") e em vários países da Europa (chamados "live births notifications"), pois se reconhece a utilidade dos dados obtidos por meio desses instrumentos no monitoramento de muitos problemas de saúde, inclusive os DC. Entretanto, frequentemente a confiabilidade das informações é questionada, e, de modo geral, se reconhece também a necessidade de investimento nos processos de geração desses dados ${ }^{16-27}$.

Confiabilidade é uma qualidade que expressa o grau de confiança que os usuários do sistema de informação têm nos dados; é, portanto, um indicador subjetivo que implica em reprodutibilidade da informação e representação dos dados de maneira consistente, sem ambiguida$\mathrm{de}^{28}$. Normalmente, a confiabilidade de um sistema de informação está associada à adequação do seu uso para determinada finalidade (quanto mais o sistema é usado com sucesso, mais as informações são registradas) e ao grau de aderência entre a informação registrada no sistema e a mesma oferecida no mundo real (concordância entre os mundos empírico e teórico do siste- ma de informação $)^{29}$. Erros em instrumentos de coleta, como as DNV e as DO, são as principais causas de equívocos nos sistemas de informação de estatísticas vitais do país. Esses erros podem ser de completeza, acurácia e precisão $0^{28}$.

\section{Completeza}

Completeza (ou completude) diz respeito ao grau de existência da informação no sistema ${ }^{28}$. Estudos nacionais de avaliação da qualidade das informações mostram que o SINASC apresenta boa completeza para a maior parte dos dados presentes na DNV, mas não para o Campo 34. Revisão dos dados do SINASC do Município do Rio de Janeiro dos anos de 1999 a 2001 mostrou frequência de $11,7 \%$ de não informação sobre $\mathrm{DC}^{30}$. Estudo caso-controle de base populacional, realizado com dados dos anos de 2000 e 2001 da região sul do Município de São Paulo, mostrou que a variável "presença de anomalia congênita" esteve ausente no SINASC em cerca de 30\% de casos e controles ${ }^{31}$.

Análises de dados mais atuais mostram uma melhora no grau de preenchimento do Campo 34 da DNV. O Município do Rio de Janeiro registrou queda do número de casos ignorados de $20,2 \%$ em 2003 para 9,3\% em 2004, à custa do aumento do número de nascidos vivos sem DC, sugerindo que os casos que constavam como ignorados eram, na verdade, recém-nascidos saudáveis ${ }^{7}$. Essa hipótese concorda com os resultados do presente estudo, pois a revisão dos prontuários dos recém-nascidos cujo Campo $34 \mathrm{da}$ DNV foi assinalado como "ignorado" ou não informado, não acrescentou muito na identificação dos DC; apenas 4 dos 59 casos identificados $(6,78 \%)$ o foram por meio dessa revisão, indicando que, no Município de São Carlos, as crianças com DC têm, na maioria das vezes, o Campo 34 das suas DNV assinalado como "não".

No Município de São Paulo, em uma coorte de 84.846 nascidos vivos durante o primeiro semestre de 2006, 12,8\% apresentaram o Campo 34 das suas DNV assinalado como "ignorado" ou em branco (não preenchido) ${ }^{11}$. Para orientar o preenchimento do Campo 34 no Município de São Paulo, em 2008 foi criado, por meio de parceria entre a Coordenação de Epidemiologia e Informação da Secretaria Municipal de Saúde e o Centro de Genética Médica da Universidade Federal de São Paulo, o projeto "Qualificação do Preenchimento do Campo 34 da Declaração de Nascido Vivo - Malformação Congênita e/ou Anomalia Cromossômica, no Município de São 
Paulo". Com base nesse projeto, uma intervenção educativa nos berçários de quatro maternidades, que contemplou inclusive avaliação à distância de casos clínicos por médicos geneticistas utilizando a Internet, conseguiu reduzir o número de DNV sem informação e ignoradas de $12,79 \%$ em 2004 para $0,76 \%$ em $2008^{12}$.

\section{Acurácia}

Acurácia - cuidado, exatidão - diz respeito ao grau de correção sintática do dado/informação e também à sua semântica ${ }^{28}$. Acredita-se que a maioria dos DC é reconhecida na criança nas primeiras horas de vida pelos profissionais da saúde, sendo o principal problema o registro da informação no próprio Campo 34 da DNV e sua codificação de acordo com a CID-10 ${ }^{12}$. Em uma maternidade de Ribeirão Preto, São Paulo, a comparação entre 6.418 DNV e prontuários médicos, comprovou que mais de $60 \%$ dos casos de DC diagnosticados não foram notificados nas $\mathrm{DNV}^{9}$.

A DNV comumente é preenchida pelo pediatra, por algum membro da equipe de enfermagem da sala de parto ou do berçário, ou por outra pessoa previamente treinada para tal fim. Não é obrigatório que seu preenchimento seja feito por profissional de saúde, sendo esta tarefa frequentemente delegada a funcionários técnicoadministrativos do estabelecimento de saúde. Problemas relacionados à transcrição da descrição do DC do prontuário para DNV são comuns, especialmente nas situações nas quais as DNV são preenchidas por profissionais não familiarizados com a terminologia médica ou com acesso restrito ao prontuário ${ }^{13}$.

A Declaração de Nascido Vivo é clara na sua pergunta: "Detectada alguma malformação congênita e/ou anomalia cromossômica?"; existem três opções de resposta: "sim", "não" ou "ignorado", diante de resposta positiva, o defeito deve ser descrito, sucintamente, em uma única linha. A pergunta aberta, sem um grupo de anomalias previamente determinadas, permite o registro de qualquer tipo de condição, evitando que DC pouco comuns não sejam notificados, mas também aumenta a complexidade do preenchimento do documento. O próprio manual de preenchimento da DNV do Ministério da Saúde não estabelece nenhuma definição de "malformação congênita e/ou anomalia cromossômica". Os responsáveis pelo preenchimento podem ter dúvidas se, por exemplo, anomalias menores ou erros inatos do metabolismo devem ser incluídos, o que contribui para subnotificação.
Uma vez que o DC esteja registrado na DNV, um outro ponto diz respeito ao processo de codificação desse defeito, realizado nos Centros de Vigilância Epidemiológica dos municípios. Nos casos de múltiplos defeitos, o fato de somente uma única anomalia poder ser escolhida para a codificação e também a falta de normas operacionais explícitas, faz com que o código da CID-10 a ser escolhido dependa de características prosaicas como, por exemplo, qual defeito foi descrito em primeiro lugar; o que impede generalização das informações e posterior comparação das mesmas dentro e fora do município.

$\mathrm{Na}$ tentativa de diminuir os erros de acurácia, os Estados Unidos introduziram no birth certificate uma lista pré-determinada de anomalias que deveriam ser registradas de forma igualitária. Porém, pesquisas realizadas antes e após a introdução dessa lista, não encontraram melhora significativa na sensibilidade do birth certificate para detectar $\mathrm{DC}^{19,21,25}$. Aumento na sensibilidade do birth certificate na notificação de DC foi constatado após a criação de um formulário específico para anomalias congênitas no prontuário do recém-nascido e a designação de apenas um profissional capacitado, por hospital, para a revisão do prontuário e o preenchimento dessa variável no certificado ${ }^{20}$.

Cursos de capacitação para o pessoal envolvido na tarefa de preencher a DNV, a elaboração de manuais que orientem o correto preenchimento do Campo 34 e o estabelecimento de critérios para codificação das anomalias registradas, são iniciativas que podem contribuir para redução da subnotificação dos DC, como demonstrou recente experiência realizada no Município de São Paulo $^{12}$.

\section{Precisão}

Precisão refere-se à concordância do dado registrado com a realidade, considerando que a diferença não ocorra por causa de erros de acurácia ${ }^{28}$. Em relação ao Campo 34 das DNV, erros de precisão aparecem principalmente nas situações nas quais há duvidas diagnósticas no momento do preenchimento da DNV (e, habitualmente, a dúvida está explícita nos prontuários, mas dificilmente é registrada nas DNV) e nas situações nas quais o DC não é visível ou é considerado como sem repercussão clínica e, portanto, é ignorado por quem preenche a DNV.

Estudo comparando a validade dos diagnósticos de DC do SINASC, em oito hospitais de sete municípios do Brasil, totalizando 53.850 nasci- 
dos vivos, verificou que o perfil de notificação não melhorou significativamente entre os anos de 2004 e $2007^{13}$. Em Nova Iorque, a criação, em 1982, de um registro específico para monitoramento dos DC, conhecido como Congenital Malformations Registry (CMR), permitiu realizar análise de dados sobre 10.882 recém-nascidos portadores de anomalias entre os anos de $1996 \mathrm{e}$ 2001, demonstrando que os birth certificates apresentam um índice de falso negativo de $29,4 \%{ }^{27}$. No presente trabalho, ficou explícito que as DNV deixam de registrar a maior parte dos casos de DC, sejam eles letais ou não. Acontece que os DC letais serão identificados posteriormente por meio das DO, de forma que o cruzamento do SINASC e do SIM permite um monitoramento mais adequado desses casos. O mesmo não ocorre em relação aos DC não letais, que permanecem subnotificados. A não identificação dos DC com alto fitness prejudica o planejamento de ações em saúde e a consequente intervenção em termos preventivos, por intermédio de aconselhamento genético para as famílias.

Diante do desconhecimento da importância do correto preenchimento da DNV para o planejamento em saúde, muitas vezes o Campo 34 da DNV é assinalado como "não" porque o responsável pelo preenchimento tem receio de registrar um defeito ainda não confirmado ou considera que, ao registrar a anomalia no documento, pode contribuir para estigmatização da criança. Luquetti et al., em 2009, já tinham percebido isso, quando, em pesquisa realizada em hospital de Campinas, São Paulo, demonstraram que dificilmente a trissomia do cromossomo 21 era registrada na DNV por meio da expressão "Síndrome de Down", embora frequentemente as dismorfias clássicas presentes nos pacientes estivessem descritas nas declarações ${ }^{10}$. Os achados do presente estudo corroboram essa percepção, pois muitas vezes havia descrição evidente de DC no prontuário e o Campo 34 da DNV era assinalado como "não". Reforçou-se a impressão, já descrita por outros autores ${ }^{8,10,27}$, de que o SINASC, por meio das DNV, registra os casos DC visíveis, que são facilmente detectados, independente da sua gravidade, como os defeitos de fechamento do tudo neural ou de membros. Enquanto o SIM, por meio das DO, registra os casos letais, representados por situações de defeitos múltiplos (síndromes cromossômicas) ou únicos com grande repercussão clínica (cardiopatias, hérnias diafragmáticas, defeitos renais), que muitas vezes não são registrados nas DNV porque os responsáveis pelo preenchimento do documento não reconhecem o defeito ou julgam, arbitrariamente, quais defeitos e em quais circunstâncias deve ou não ser feita a notificação.

Além do problema da qualidade da informação que é registrada nas DNV, o presente estudo demonstrou incongruências entre os dados divulgados pelo DATASUS e os dados que estão arquivados na Vigilância Epidemiológica (VE) do Município de São Carlos. A discordância numérica de informações ente os dois bancos de dados não faz sentido, porque os dados publicados pelo DATASUS deveriam ter sido gerados a partir das DNV. Isso denota problemas na alimentação do SINASC no Município de São Carlos, que podem ter ocorrido durante a atualização dos dados, bem como em função de processos de mudança e reestruturação do próprio sistema. Esses problemas estão relacionados a erros na digitação, exclusão de dados já digitados, falhas de hardware e ausência de rotinas de realização de cópias de segurança, com perda de bancos de dados.

\section{Considerações finais}

Erros de acurácia e precisão, relacionados principalmente à transcrição da descrição do DC do prontuário para DNV e a codificação e alimentação do SINASC, comprometeram a confiabilidade das DNV como fonte de informações sobre os DC no Município de São Carlos, entre os anos de 2003 e 2007. A escassez de dados oficiais no Brasil referentes aos DC aponta para a necessidade do aperfeiçoamento dos sistemas de informação já existentes. Para o aprimoramento do SINASC é fundamental o envolvimento das Secretarias $\mathrm{Mu}-$ nicipais de Saúde, visto ser esse o local de codificação e de alimentação do sistema, mas também é importante o maior envolvimento dos estabelecimentos de saúde, com capacitação dos profissionais que preenchem a DNV. Com orientação sobre a importância e o funcionamento da DNV, o SINASC pode se tornar um excelente sistema de monitoramento e vigilância para os defeitos congênitos. As bases de dados do SIM e do SINASC podem ainda ser vinculadas, o que aumenta a sensibilidade de deteç̧ão, permitindo que os municípios se planejem para atenderem corretamente às crianças com anomalias, dimensionando suas necessidades e elaborando um programa de prevenção às diversas etiologias. 


\section{Colaboradores}

GC Nhoncanse participou da coleta, análise e interpretação dos dados, além da redação do artigo. DG Melo participou da análise e interpretação dos dados, organização e redação do artigo, revisão e edição final.

\section{Agradecimentos}

À Secretaria Municipal de Saúde de São Carlos e à Santa Casa de Misericórdia de São Carlos, por permitirem a consulta aos documentos. À Sra. Rosana Trigo, da Vigilância Epidemiológica do Município de São Carlos, e à Sra. Renata Olaia Nunes, da Santa Casa de Misericórdia de São Carlos, pela ajuda operacional durante a coleta dos dados. À Fundação de Amparo à Pesquisa do Estado de São Paulo (FAPESP), pelo apoio financeiro.

\section{Referências}

1. Brasil. Ministério da Saúde (MS). Manual de Instruções para o preenchimento da Declaração de Nascido Vivo. $3^{\text {a }}$ ed. Brasília: Ministério da Saúde, Fundação Nacional de Saúde (MS); 2001.

2. Organização das Nações Unidas (ONU). Organização Mundial de Saúde (OMS). Organização PanAmericana da Saúde (OPAS). Prevenção e controle de enfermidades genéticas e defeitos congênitos: relatório de um grupo de consulta. Washington DC: Organização Pan-Americana da Saúde (OPAS); 1984.

3. Marques-de-Faria AP, Ferraz VE, Acosta AX, Brunoni D. Clinical genetics in developing countries: the case of Brazil. Community Genet 2004; 7(2-3):95105.

4. Horovitz DDG, Llerena Júnior. JC, Mattos RA. Atenção aos defeitos congênitos no Brasil: panorama atual. Cad Saude Publica 2005; 21(4):1055-1064.

5. Horovitz DDG, Cardoso MHCA, Llerena Jr. JC, Mattos RA. Atenção aos defeitos congênitos no Brasil: características do atendimento e propostas para formulação de políticas públicas em genética clínica. Cad Saude Publica 2006; 22(12):2599-2609.

6. Brasil. Ministério da Saúde. Departamento de Informação e Informática do SUS. Sistema de Informações de Saúde. Estatísticas Vitais - Mortalidade e Nascidos Vivos. 1979-2007. [acessado 2011 jan 07]. Disponível em: http://www.datasus.gov.br

7. Guerra FAR, Llerena JC, Gama SGN, Cunha CB, Theme Filha MM. Defeitos Congênitos no Município do Rio de Janeiro, Brasil: uma avaliação através do SINASC (2000-2004). Cad Saude Publica 2008; 24(1):140-149.

8. Guerra FAR, Llerena JC, Gama SGN, Cunha CB, Theme Filha MM. Confiabilidade das informações das declarações de nascido vivo com registro de defeitos congênitos no Município do Rio de Janeiro, Brasil, 2004. Cad Saude Publica 2008; 24(2):438446.

9. Righeto ALC, Huber J, Machado JC, Melo DG. Anomalias congênitas: validade das informações das declarações de nascido vivo em uma maternidade de Ribeirão Preto, São Paulo. Pediatria (São Paulo) 2008; 30(3):159-164.

10. Luquetti DV, Koifman RJ. Quality of reporting on birth defects in birth certificates: case study from a Brazilian reference hospital. Cad Saude Publica 2009; 25(8):1721-1731.

11. Geremias AL, Almeida MF, Flores LPO. Avaliação das declarações de nascido vivo como fonte de informação sobre defeitos congênitos. Rev Bras Epidemiol 2009; 12(1):60-68. 
12. De Nicola PDR, Cernach MCSP, Perez ABA, Brunoni D. A utilização da Internet na notificação dos defeitos congênitos na Declaração de Nascido Vivo em quatro maternidades públicas do Município de São Paulo, Brasil. Cad Saude Publica 2010; 26(7):13831390.

13. Luquetti DV, Koifman RJ. Qualidade da notificação de anomalias congênitas pelo Sistema de Informações sobre Nascidos Vivos (SINASC): estudo comparativo nos anos 2004 e 2007. Cad Saude Publica 2010; 26(9):1756-1765.

14. Organização das Nações Unidas. Programa das Nações Unidas para o Desenvolvimento. Atlas do Desenvolvimento Humano no Brasil. 2002. [acessado 2011 jan 07]. Disponível em: http://www.pnud. org.br/idh.

15. Estado de São Paulo. Secretaria de Economia e Planejamento. Fundação Sistema Estadual de Análise de Dados (SEADE). Taxas de Mortalidade Infantil. 2009. [acessado 2011 jan 07]. Disponível em: http:/ /www.seade.gov.br/produtos/mortinf/tabelas/2009/ pdf/tabela02_2009.pdf.

16. Hay S, Lunde AS, Mackeprang M. Background and Methodology of a Study of Congenital Malformations. Public Health Reports 1970; 85(10):913-918

17. Mackeprang M, Hay S, Lunde AS. Completeness and Accuracy of Reporting of Malformations on Birth Certificates. HSMHA Health Rep 1972; 87(1):43-49.

18. Naylor A, Eaton AP, Aplin ER, Eska B. Birth Certificate Revision and Reporting of Congenital Malformations. Am J Public Health 1974; 64(8):786-791.

19. Frost F, Starzyk P, George S, McLaughlin JF. Birth complication reporting: the effect of birth certificate design. Am J Public Health 1984; 74(5):505-506.

20. Minton SD, Seegmiller RE. An improved system for reporting congenital malformations. JAMA 1986; 256(21):2976-2979.

21. Hexter AC, Harris JA. Bias in congenital malformations information from the birth certificate. Teratology 1991; 44(2):177-180.

22. Snell LM, Little BB, Knoll KA, Johnston WL Jr, Rosenfeld CR, Gant NF. Reliability of birth certificate reporting of congenital anomalies. Am J Perinatol 1992; 9(3):219-222.

23. Piper JM, Mitchel Jr. EF, Snowden M, Hall C, Adams M, Taylor P. Validation of 1989 Tennessee birth certificates using maternal and newborn hospital records. Am J Epidemiol 1993; 137(7):758-768.

24. Hemminki E, Meriläinen J, Teperi J. Reporting of malformations in routine health registers. Teratology 1993; 48(3):227-231.
25. Watkins ML, Edmonds L, McClearn A, Mullins L, Mulinare J, Khoury M. The Surveillance of Birth Defects: The Usefulness of the Revised US Standard Birth Certificate. Am J Public Health 1996; 86(5):731734.

26. Wang Y, Sharpe-Stimac M, Cross PK, Druschel CM, Hwang SA. Improving case ascertainment of a population-based birth defects registry in New York State using hospital discharge data. Birth Defects Res A Clin Mol Teratol 2005; 73(10):663-638.

27. Wang Y, Druschel CM, Cross PK, Hwang SA, Gensburg LJ. Problems in using birth certificate files in the capture-recapture model to estimate the completeness of case ascertainment in a populationbased birth defects registry in New York State. Birth Defects Res A Clin Mol Teratol 2006; 76(11):772-777.

28. Silva JPL, Travassos C, Vasconcellos MMC, Campos LM. Revisão sistemática sobre encadeamento ou linkage de bases de dados secundários para uso em pesquisa em saúde no Brasil. Cad Saúde Colet 2006; 14(2):197-224.

29. Martins GA. Sobre Confiabilidade e Validade. RBGN 2006; 8(20):1-12.

30. Theme Filha MM, Gama SGN, Cunha CB, Leal MC. Confiabilidade do Sistema de Informações sobre nascidos vivos hospitalares no Município do Rio de Janeiro, 1999-2001. Cad Saude Publica 2004; 20(Supl.1):83-91.

31. Almeida MF, Alencar GP, França Jr. I, Novaes HMD, Siqueira AAF, Schoeps D, Campbell O, Rodrigues LC. Validade das informações das declarações de nascidos vivos com base em estudo de caso-controle. Cad Saude Publica 2006; 22(3):643-652.

Artigo apresentado em 15/03/2011

Aprovado em 25/03/2011

Versão final apresentada em 30/06/2011 